cevons à la base des escarpements de la rive gauche, sur les raides éboulis calcaires qui leur font suite, non pas un pied, mais des centaines de pieds d'A. Moly en pleine floraison, dont les touffes serrées, surchargées d'ombelles d'un jaune doré, formaient lè plus brillant tapis qu'on puisse imaginer. Qu'on juge de notre joie et si les échos de la montagne retentirent longtemps de nos vivats en l'honneur de Pourret!

Le doute n'était plus possible. Cette station située en pleine montagne, à 550 mètres d'altitude et à plusieurs kilomètres des cultures et des jardins de la plaine, oủ d'ailleurs, ainsi que nous nous en sommes assurés, notre espèce est absolument inconnue, ne pouvait être que naturelle. S'agirait-il ici d'une tentative de naturalisation couronnée de succès? Cela paraît tout à fait improbable, car en ce point le sol, dont la terre végétale se cache sous une épaisse couche de débris calcaires que les hampes et les feuilles de notre Ail ne traversent qu'avec assez de peine pour arriver à la lumière, semble peu propre à être choisi pour une expérience de ce genre.

Quelques jours plus tard, au reste, un nouveau fait se produisit, qui devait enlever tous les doutes. M. G. Gautier, en explorant à nouveau la coumo de l'Aigo, voisine de celle des Baux, fut assez heureux pour y trouver notre espèce en abondance et en sept stations différentes.

Nous pouvons donc inscrire à nouveau, mais cette fois en toute certitude, l'A. Moly L. dans la flore française avec les indications suivantes:

Montagne d'Alaric, près Moux (Aude), éboulis calcaires exposés au midi de la partie supérieure des ravins ou coumos des Baux et de l'Aigo, altitude 500 mètres environ (Pourret), très abondant.

C'est une plante de plus à ajouter à celles de la France, et un nouveau témoignage rendu à la sagacité et à la véracité du grand botaniste dont la mémoire nous est si chère.

\title{
QUELQUES JOURS D'HERBORISATIONS AUTOUR D'AX (ARIÉGE),
} par M. D. CHoS.

Appelé à séjourner à Ussat-les-Bains aux mois d'août 1860 et de septembre 1861, je cherchai à dresser la liste des plantes les plus importantes observées par moi en ce point d'une des principales vallées de l'Ariége, et l'Esquisse de la végétation d'Ussat parut en 1862, dans la Revue des Sociétés savantes, I, p. 313-320. Bientôt après, le $\mathrm{D}^{\mathrm{r}}$ Guitard étendit cette liste, soit dans son Guide $\dot{a}$ Ussat-les-Bains (1863), p. 109 et suiv., soit dans ses Souvenirs d'Ussat (1865), p. 159 et suiv.

Ayant résidé durant une quinzaine de jours du mois d'aoùt dernier dans la petite ville d'Ax, située dans la même vallée, mais à 23 kilomètres au 
sud d'Ussat et à 720 mètres d'altitude (tandis qu'Ussat n'est qu'à 450 mètres, au niveau du lit de l'Ariége), j'ai tenu d'autant plus à faire connaissance avec la végétation du lieu, qu'une différence tranchée dans la nature des roches sépare ces deux points de la vallée: là je l'avais trouvée formée par deux pans de montagnes calcaires, ici l'un d'eux est granitique et l'autre schisteux; il y avait donc, indépendamment des différences de hauteur, un curieux parallèle à établir entre les flores des deux stations thermales, rendu plus intéressant encore par suite des récents travaux de Weddell et de M. Contejean sur l'influence de la nature du sol dans la répartition des plantes. D'ailleurs la localité d'Ax a été à peine explorée par les naturalistes (1). J'ai donc profité de tous mes loisirs pour recueillir ou noter lès espèces qui se sont offertes à moi dans des promenades autour de la ville et dans de nombreuses excursions sur les hauteurs des montagnes voisines ; néanmoins ma liste sera sûrement bien incomplète par l'effet, soit de la brièvetė du séjour, soit de la sécheresse intense qui avait brûlé un certain nombre de plantes et nui au développement de plusieurs: une riche moisson attend donc encore le botaniste qui pourra se livrer à des investigations suivies sur les productions de cette vallée de l'Ariége.

Les vieux murs de la ville d'Ax portent :

Asplenium Ruta-muraria L.

Veronica arvensis L.

- didyma $L$.

Linaria minor Desf.
Campanula Erinus L. Sedum dasyphyllum $\mathrm{L}$. Umbilicus pendulinus DG.

et de plus ceux qui avoisinent un petit pont au confluent de l'Ariége et de l'Oriége :

Antirrhinum Asarina L.

Saxifraga hirsuta $\mathrm{L}$.

Arabis alpina L.
Cheiranthus Cheiri L.

Saponaria officinalis $\mathbf{L}$.

Tout autour de la ville on peut relever ce stock si nombreux de plantes éminemment triviales en tous lieux, et signalées uniquement ici en faveur de ceux qui auront à cœur de retrouver les espèces mentionnées par nous ou de compléter cette ébauche de florule locale :

(1) Cependant cette contrée a été parcourue par Lapeyrouse, qui a écrit : "Anno 1776... exploratis depressioribus locis, circa Tarascon et thermas Ussat, ad aliud iter nos paravimus. Urbem Ax invisimus, pagum Mœrens conscensi, prata alpina, iter faciendo, non sine magno plantarum proventu, percurrimus..." (Voy. Quelques pages inédites de Lapeyrouse, in Mém. Acad. des sc. de Toulouse, $5^{e}$ série, t. IV, p. 518-523.)

J'ai appris qu'une liste comprenant 91 espèces de plantes d'Ax avait été dressée par le $\mathrm{D}^{r}$ Seriès, ancien inspecteur des eaux thermales de ce lieu; mais je n'ai pas pu me la procurer. 
Polypodium vulgare L.

Ceterach officinarum Willd.

Hordeum murinum L.

Poa annua $\mathrm{L}$.

Lolium perenne L.

Plantago lanceolata L.

- media L.

- major L.

Mentha rotundifolia $\mathrm{L}$.

- silvestris $L$.

Convolvulus arvensis $\mathrm{L}$.

Solanum nigrum L.

Hypochœris radicata $\mathrm{L}$.

Crepis diffusa DG.

Taraxacum officinale Wigg.

Sonchus oleraceus L.

- asper Vill.

Lapsana communis $\mathrm{L}$.

Lappa minor DC.

Cirsium lanceolatum Scop.

Onopordon Acanthium L.

Centaurea Cyanus L.

Carlina vulgaris $\mathrm{L}$.

Achillea Millefolium L.

Anthemis Cotula L.

Bellis perennis L.

Erigeron canadensis L.

Senecio vulgaris $\mathbf{L}$.

Rubus fastigiatus Weihe.

et dans les fossés :

Equisetum arvense L.

- palustre L.

Juncus conglomeratus L.

- effusus L.

- bufonius L.

- - var. fasciculatus Bert.

Glyceria fluitans R. Br.

Veronica Anagallis $\mathrm{L}$.

- Beccabunga $\mathbf{L}$.
Rubus discolor Weihe.

- cæsius L.

Medicago Lupulina L.

Trifolium repens $\mathrm{L}$.

- pratense L.

- campestre Schreb.

Malva rotundifolia $\mathrm{L}$.

- silvestris L.

Oxalis corniculata L.

Erodium cicutarium L'Hérit.

Portulaca sativa L.

Linum usitatissimum $\mathrm{L}$.

Stellaria media Vill.

Cerastium vulgatum $\mathrm{L}$.

Fumaria officinalis L.

Raphanus Raphanistrum L.

Sisymbrium offieinale Scop.

Capsella Bursa-pastoris L.

Chenopodium album $\mathrm{L}$.

- Vulvaria L.

Rumex pulcher L.

- obtusifolius $\mathbf{L}$.

Amarantus retroflexus $\mathrm{L}$.

- silvestris $\mathbf{L}$.

Chenopodium hybridum L.

- opulifolium Schrad.

- Mercurialis annua L.

Urtica dioica $\mathrm{L}$.

Parietaria diffusa M. et Koch.

On distinguera encore à quelques pas d'Ax, comme espèces communes :

Asplenium septentrionale L.

- Trichomanes L.

- Adiantum nigrum L.

Polystichum Filix-mas Roth.

Cynosurus echinatus $\mathbf{L}$.

Bromus maximus Desf.
Gnaphalium uliginosum ${ }^{`} \mathrm{~L}$.

Inula dysenterica L.

Galium palustre L.

Epilobium hirsutum L.

Ranunculus repens L.

- acris L.

- hederaceus L.

Stellaria uliginosa Murr. 
Marrubium vulgare L.

Calamintha Nepeta Link.

Origanum vulgare $\mathrm{L}$.

Teucrium Scorodonia L.

Verbascum Lychnitis L.

- floceosum W. et Kit.

- lychnitidi-floccosum Kit.

Brunella vulgaris $L$.

Galeopsis Ladanum L.

- - angustifolia Ehrh.

Linaria striata DC.

Lycium barbarum L.

Calystegia sepium R. Br.

Campanula rotundifolia L.

- Trachelium I.

Jasione montana $\mathrm{L}$.

Barkhausia fœtida DC.

Picris hieracioides L.

Andryala integrifolia L.

Lactuca virosa $\mathbf{L}$.

Tolpis barbata Cass.

Senecio adonidifolius Lois.

Artemisia vulgaris $L$.

- Absinthium L.

Filago arvensis L.

- germanica $\mathrm{L}$.

Cirsium eriophorum Scop.

Anthemis arvensis L.

Scabiosa Columbaria l.

Knautia arvensis L.

Tordylium maximum $\mathbf{L}$.
Potentilla argentea L.

- verna $L$.

Trifolium striatum L.

-- arvense L.

Sedum brevifolium DE.

— hirsutum All.

-. dasyphyHum L.

- reflexum $\mathrm{L}$.

Hypericum perforatum L.

- tetrapterum Fries.

Circæa lutetiana $\mathrm{L}$.

Geum urbanum $\mathrm{L}$.

Malva moschata $\mathrm{L}$.

Lychnis dioica $\mathrm{L}$.

- Githago Lamk.

Silene inflata Sm.

- gallica L.?

Dianthus Carthusianorum L.

Torilis Anthriscus Gmel.

Daucus Carota L.

Fœniculum vulgare Gærtn.

Gypsophila muralis L.

Irenaria serpyllifolia $\mathbf{L}$.

Sagina procumbens $\mathbf{L}$.

- apetala $\mathbf{L}$.

Msine tenuifolia Crantz.

spergularia rubra Pers.

Scleranthus annuus L.

- perennis L.

Bunias Erucago L.

Sisymbrium Alliaria Scop.

Quelques-unes des espèces qu'on vient de citer m'ont paru vraiment caracléristiques par leur fréquence même; ce sont :

Teucrium Scorodonia L.

Verbascum Lychnitis L.

- floccosum Waldst. et Kit.

- Iychnitidi-floccosum Kit.

Senecio adonidifolius Lois.

Andryala integrifolia L.
Lactuca virosa $\mathrm{L}$.

Campanula rotundifolia L.

Potentilla argentea L.

Sedum Fabaria Koch.

- reflexum $\mathrm{L}$.

Sarothamnus purgans Godr. Gren.

Cette dernière plante, qui autour de la ville tapisse abondamment les rochers, où elle se détache par sa teinte d'un vert glauque, mariant presque toujours ses rameaux raides à ceux du Genèt à balais ou aux feuilles de la Grande-Fougère, y remplace l'Ajonc, dont on cherche en vain la moindre trace, et s'èlève jusqu'à 2000 mètres, car je l'ai observée près du pic du Saquet.

Intéressante aussi à étudier est la Potentille argentée, qui accom- 
pagne le touriste, de la ville d'Ax jusqu'aux plus grandes hauteurs des environs.

Je suis frappé de ne pas avoir noté, dans mes courses, de représentant des genres suivants : Lycopodium, Scolopendrium, Soldanella, Samolus, Eryngium et Bupleurum, Aconitum, Reseda, Diplotaxis. Mais je n'ai garde d'en conclure qu'ils manquent à la flore d'Ax.

Sur les rochers qui bordent la route, près du pont d'Espagne, se montre en abondance l'Hieracium pseudo-Eriophorum Loret et Timb., aussi commun que les $H$. silvaticum Lamk et umbellatum L.; et, après avoir dépassé ce pont, un petit sentier qui se trouve à droite m'a offert, indépendamment du Viola mirabilis L., trois espèces à l'état sec et à peine reconnaissables, que je n'ai point vues ailleurs : Linaria Cymbalaria $\mathrm{L}$., Sedum Cepaa L., Saxifraga granulata L.

A peine fait-on quelques pas sur la montagne qu'on rencontre :

Polypodium rhæticum $\mathrm{L}$.

Briza media L.

Bromus mollis L.

Poa pratensis L.

Dactylis glomerata L.

Cynosurus cristatus L.

Holcus mollis L.

Carex muricata $\mathbf{L}$.

- Jeporina L.

Scirpus setaceus L.

Allium sphærocephalum L.

Brunella grandiflora $\mathrm{L}$.

Stachys recta $\mathrm{L}$.

Thymus Serpyllum L.

Calamintha Acinos Clairv.

Galeopsis Tetrahit L.

Lamium maculatum $\mathrm{L}$.

Echium vulgare $\mathrm{L}$.

Orobanche Rapum Th.

Erythræa Centaurium Pers.

- pulchella Horn.

Hieracium umbellatum L.

- silvaticum L.

Thrincia hirta Roth.

Leontodon hispidus $\mathrm{L}$.

- pyrenaicus Gou.

Picris hieracioides $\mathbf{L}$.

Carlina vulgaris L.

Cirsium palustre Scop.

Eupatorium cannabinum L.

Tussilago Farfara L.
Solidago Virga-aurea L.

Leucanthemum vulgare Lamk.

Senecio Jacobæa L.

Artemisia campestris L.

Campanula patula $\mathrm{L}$.

- Trachelium L.

- glomerata L.

Scabiosa Succisa L.

Knautia dipsacifolia Host.

Valeriana officinalis L.

Galium verum $\mathrm{L}$.

- silvestre Poll.

- elatum Thuill.

- Cruciata Scop.

- vernum Scop.

Pimpinella Saxifraga L.

- magna $L$.

Peucedanum Oreoselinum Mœnch.

Chærophyllum temulum L.

$\Lambda$ ngelica pyrenæa Spreng.

Vicia sepium L.

Lotus uliginosus Schk.

- corniculatus L.

Trifolium aureum Poll.

Potentilla Tormentilla Nestl.

Poterium Sanguisorba L.

Chrysosplenium oppositifolium I.

Linum catharticum L.

- gallicum $\mathbf{L}$.

Viola alpestris Jord.

Ranunculus bulbosus L.

Se dirige-t-on vers la sapinière du Llata, on cueille d'abord : 
Silene nutans L.

- rupestris L.

Dianthus Armeria L.

- prolifer L.

Et dans les bois :

Luzula multiflora Lej.

Tamus communis $\mathrm{L}$.

Lonicera Periclymenum L.

Rhamnus Frangula L.

Ilex Aquifolium L.
Stellaria Holostea L.

- graminea $\mathbf{L}$.

- glauca With.

Euphorbia verrucosa $\mathbf{L}$.

Au hameau de Petches, Galeopsis Tetrahit L.; puis :

Deschampsia flexuosa P. Beauv.

Carex leporina L.

- divulsa Good.

Myosotis alpestris Schm.

Pedicularis silvatica $\mathrm{L}$.

Euphrasia minima DC.(E. Soyeri Timb.)

Veronica serpyllifolia L.

Pinguicula vulgaris $\mathrm{L}$.

Gentiana Pneumonanthe L.

Conopodium denudatum Koch.
Bryonia dioica L.

Salix aurita L.

- Caprea L.

Juniperus communis L.

Avant de quitter le Hêtre et d'entrer dans la sapinière, on voit le Dianthus deltoides L. venir se mêler au D. Carthusianorum L., qu'il remplace bientôt complètement; là aussi quelques pieds de Carlina caulescens Lamk prennent place au milieu de ceux du C. acanthifolia All.

L’entrée de la forêt de Sapin argenté (Abies pectinata DC.) montre :

Blechnum Spicant Roth.

Luzula nivea DC.

Vaccinium Myrtillus L.
Trifolium spadiceum L. Spiræa Aruncus L.

Saxifraga leucanthemifolia L.

Drosera rotundifolia L.

Parnassia palustris L.

Polygala vulgaris $L$.

Epilobium alpinum L.

Hepatica triloba Chaix.

Biscutella saxatilis Schleich.

Sur la route qui conduit au hameau des Bazerques j’ai cueilli :

Cyperus flavescens L.

Lysimachia Nummularia L.

| Gypsophila muralis L.

et le long du chemin qui relie Ax à Mérens :

Anagallis tenella $\mathrm{L}$.

Erigeron acris L.

Rhododendron ferrugineum L.

Genista sagittalis L.

Rumex Acetosella L.

Suivons la nouvelle route d'Ax à Orgeix (village à 3 kilomètres d'Ax, altitude 809 mètres), et nous y signalerons, entre autres espèces :

Senecio viscosus $\mathrm{L}$.

Gnaphalium luteo-album L.

Leontodon autumnalis $\mathrm{L}$.
Lathyrus silvestris $\mathrm{L}$.

Nasturtium pyrenaicum R. Br.

Quercus Toza Bosc. 
et au village d'Orgeix :

Juncus Gerardi Lois.

CaJamintha Nepeta Link.

Glechoma hederacea L.

Galeobdolon luteum L.

Mentha arvensis $\mathbf{L}$.

- sativa L.

Bidens tripartita $\mathbf{L}$.

Sherardia arvensis L.

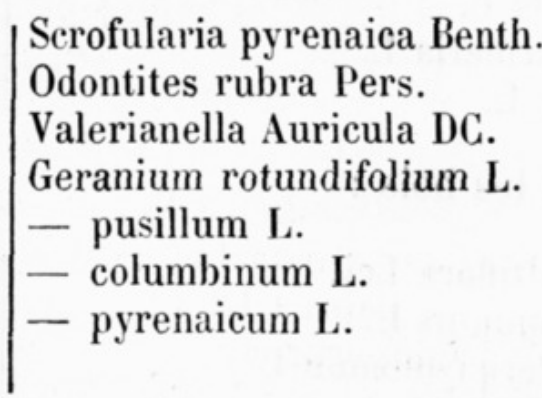

Si du village d'Orgeix on gagne celui d'Orlu (altit. 824 mètres), on rencontrera dans les marais que l'on còtoie :

Potamogeton fluitans Roth.

Cyperus flavescens $\mathrm{L}$.

Juncus lamprocarpus Ehrh.

Veronica anagalloides Guss.

Bidens tripartita L.

- cernua L.

Nasturtium officinale R. Br.

et sur le bord du chemin, Astragalus glycyphyllos L.

Poussons jusqu'aux forges d'Orlu, limite extrème de la vallée, et après avoir été ravi par la beauté et la fraîcheur du site, par les magnifiques cascades qui tombent de tous côtés, on pourra récolter :

Pulmonaria saccharata Mill.

Solidago Virga-aurea L.

Prenanthes muralis L.

- purpurea L.

Phyteuma spicatum L.

Trifolium patens Schreb.

Orobus luteus L.

Rubus idæus L.
| Geranium silvaticum L. Cardamine hirsuta L. Erucastrum obtusangulum Rchb. Thalictrum aquilegifolium $\mathbf{L}$. Dianthus deltoides L. Euphorbia hiberna L. - silvatica $\mathrm{L}$.

Pendant le retour par la montagne, en contournant le parc du château d'Orgeix :

Polystichum Filix-mas Roth.

Asplenium Filix-femina L.

Tamus communis $\mathrm{L}$.

Stachys silvatica $L$.

Veronica Chamædrys L.

- officinalis L.

Lysimachia nemorum $\mathrm{L}$.

\author{
Scabiosa Succisa L. \\ Gnaphalium silvaticum L. \\ Rhamnus Frangula I. \\ Helleborus viridis $\mathbf{L}$. \\ Viola cornuta L. \\ Clematis Vitalba $L$.
}

A moins de 2 kilomètres au-dessous d'Ax, et dans la même vallée, est le village de Savignac, de chaque côté duquel la montagne offre deux ravins encaissés dans d'étroits vallons que le botaniste aura tout intérêt à explorer; l'un deux, celui qui est sur la rive gauche de l'Ariége, frappe de loin et attire le voyageur par une belle cascade que l'on aperçoit de la grande route. 
Après avoir remarqué dans le village : Centaurea Calcitrapa L., Bidens tripartita L., et dans les prairies d'alentour : Deschampsia ccespitosa $\mathrm{P}$. Beauv., Mentha sativa L., Pulmonaria saccharata Mill., Hieracium Pilosella L., Angelica Razoulii Lap., Agrimonia Eupatoria L., Lythrum Salicaria L., Polygonum Bistorta L., il verra paraitre, en s'élevant dans les pâturages marécageux :

Carex stellulata L.

- glauca Scop.

Eriophorum gracile Koch.

Narthecium ossifragum Huds.

Luzula nivea DC.

Orchis maculata $\mathrm{L}$.

Scrofularia pyrenaica Benth.

Digitalis purpurea $\mathrm{L}$.

Melampyrum pratense L.

Rhinanthus minor Ehrh.

Gentiana lutea L.

Myosotis alpestris Schl.

Pinguicula vulgaris $L$.

Mulgedium Plumieri DC.

Crepis blattarioides Vill.

- lampsanoides Frœl.

Leontodon pyrenaicus Gou.

- hispidus L.

Astrantia major L.

Carum verticillatum Koch.

- Carvi L.
Heracleum pyrenaicum Lamk.

Chærophyllum aureum L.

Genista sagittalis L.

Trifolium spadiceum L.

- alpestre L.

Vicia Orobus DC.

- sepium L.

Alchemilla vulgaris $L$.

Spirea Aruncus L.

- Ulmaria L.

Saxifraga stellaris $\mathbf{L}$.

Parnassia palustris $\mathbf{L}$.

Polygala vulgaris $\mathrm{L}$.

Geranium pyrenaicum L.

Helianthemum vulgare Pers.

Viola cornuta L.

Cardamine latifolia L.

Trollius europæus $\mathbf{L}$.

Montia rivularis Gmel.

Rumex Acetosa L.

Et, à la lisière du bois, le long de la vallée :

Solanum Dulcamara L.

Orobus tuberosus L.

Conopodium denudatum Koch.

Circæa lutetiana L.

Impatiens Noli-tangere L.

Hepatica triloba DC.

Dans la forêt qui surplombe la vallée :

Vaccinium Myrtillus L.

Rhododendron ferrugineum $\mathrm{L}$.

Veronica officinalis L.

Prenanthes purpurea L.

Gnaphalium silvaticum $\mathbf{L}$.
Asperula odorata L.

Oxalis Acetosella L.

Aquilegia (feuilles).

Anemone nemorosa $\mathrm{L}$.

Si l'on remonte le ravin de l'autre côté de la grande route et qui plus haut s'élargit en vallée contournant les villages d'Igneaux et de Sorgeat, on trouvera :

Mentha silvestris L. var. candicans Wirtg.

Antirrhinum Asarina L.

Cuscuta minor DC.

Centaurea amara L

Carduus nutans $\mathrm{L}$. 
Cirsium monspessulanum All

Eupatorium cannabinum L.

Levisticum officinale Koch.

Laserpitium latifolium $\mathrm{L}$.

Sur la montagne d'Igneaux :

Luzula campestris DC.

Petasites niveus Baumg.

Campanula rapunculoides $\mathrm{L}$.

Et en descendant de Sorgeat :

Artemisia campestris L.

Campanula glomerata L.
Hepatica triloba DC.

Cardamine latifolia Vahl.

Helianthemum vulgare Pers.

Salix purpurea $\mathbf{L}$.

Cerastium trigynum Vill. Spergularia rubra Pers.

Le 23 août, je faisais, en compagnie d'un de nos confrères, M. Fugairon, docteur ès sciences naturelles, et de mon fils, l'ascension d'un des pics élevés des environs d'Ax, le Saquet (2306 mètres), au sud-ouest de la ville, sur terrain granitique.

Après avoir gravi le pied de la montagne et rencontré en abondance dans le bois de Hêtre, avec l'Airelle Myrtille, Prenanthes purpurea, Mulgedium Plumieri, Bunium denudatum, Daphne Mezereum, D. Laureola, nous voyons succéder au Hêtre, vers 1000 mètres environ (au plateau dit de Bonascre), le Sapin argenté, parfois d'un effet si original par les perruques blanches d'un Lichen filamenteux (Cornicularia jubata DC.) pendant de ses branches.

En même temps apparaissaient Genista sagittalis dans les clairières, Luzula nivea, les Jasione perennis et humilis remplaçant le J. montana, Rhododendron ferrugineum, d'abord en pieds isolés, puis se groupant peu à peu en touffes réunies même sur certains points en massifs de plusieurs mètres de pourtour. Bien que la plupart soient en fruit, quelques-uns des plus élevés sur la montagne montrent encore leurs fleurs.

Ajoutons Chenopodium Bonus-Henricus, un Sempervivum (rosettes seules), Gentiana lutea dont quelques rares individus fleuris, Antennaria dioica Gærtn., la Sabine succédant au Genévrier commun, Juncus alpinus W., Deschampsia flexuosa P. B., Polypodium Dryopteris L.

A 1600 mètres environ, se trouve la cabane dite de Mancel, que l'on était en train de remplacer par un joli chalet, et autour de laquelle j'ai cueilli :

Carex leporina $\mathbf{L}$.

- glauca $\mathbf{I}$.

Orchis maculata L. ? (défleuri).

Lilium Martagon L. (en feuilles).
Sambucus racemosa $\mathrm{L}$.

Cardamine amara $\mathrm{L}$.

Rumex alpinus L.

Une nouvelle ascension jusqu'aux paturages surmontant la forêt et 
dominés par le pic du Saquet, au-dessous duquel des 'plaques de neige occupaient encore les crevasses des rochers, montre :

Phleum alpinum L.

Vaccinium uliginosum L., y remplaçant le $V$. Myrtillus L.

Azalea procumbens L.

Veronica fruticulosa $\mathrm{L}$.

Arnica montana L. - RR.

Carlina caulescens Lamk.

Hieracium pumilum Lap. - CC. (1).

Campanula precatoria Timb. - RR.

Potentilla pyrenaica Ram.

Alchemilla alpina $\mathrm{L}$.

Dianthus monspessulanus L.

Thesium pratense Ehrh.

Un pied de Calluna vulgaris s'offre avec des fleurs blanches.

Enfin l'ascension du pic lui-même donne :

Agrostis alpina Scop.

Nardus stricta $L$.

Festuca spadicea $\mathbf{L}$.

Phyteuma hemisphæricum L.
Meum athamanticum Jacq. Sarothamnus purgans Gr. God. Saxifraga geranioides $\mathrm{L}$.

La comparaison des flores d'Ax et d'Ussat dévoile entre ces deux stations thermales d'une même vallée les différences les plus tranchées, déterminées par la nature du sol, calcaire dans celle-ci, surtout granitique et schisteux dans celle-là ; la liste serait longue des plantes exclusivement propres à chacune d'elles. Je n'ai point rencontré à Ax un seul pied d'Erinus, de Teucrium pyrenaicum, de Globularia nana, de Centranthus angustifolius, de Genista Scorpius, de Saxifraga Aizoon, si abondants à Ussat, où manquent Senecio adonidifolius, Potentilla argentea et tant d'autres espèces signalées dans la présente esquisse.

Un champ bien intéressant d'études attend le botaniste qui pourra explorer à loisir les diverses localités comprises entre ces deux points de la vallée, suivre de l'une vers l'autre l'extension des diverses espèces caractéristiques en rapport avec la nature du sol, et marquer les limites. Il y a là de nouveaux éléments à recueillir pour la solution de cette question, toujours reprise et alternativement résolue en sens contraires, du degré d'influence, soit physique, soit chimique, du sol sur la végétation (2).

\section{G. Bainier fait à la Société la communication suivante :}

(1) Cette curieuse espèce, découverte par Lapeyrouse au sommet du Cambredases, au Canigou, à Costabona (Hist. abrég. des pl. des Pyr. 469), pais retrouvée au col de Nouri et au port de Saldeu, et aussi à Maillet (Hautes-Pyrénées), est extrêmem ent abondante dans la nouvelle localité signalée.

(2) Voyez notamment dans ce Bulletin (Séances', t. XXVI, p. 338, une note de M. Gaston Bonnier, communiquée à la Société au mois de décembre dernier et peu favorable aux conclusions de M. Contejean sur l'influence chimique du sol sur la végétation. 


\section{$2 \mathrm{BHL}$ Biodiversity Heritage Library}

Clos, Dominique. 1880. "Quelques Jours D'herborisations Autour D'ax (Ariége)." Bulletin de la Société botanique de France 27, 216-225.

https://doi.org/10.1080/00378941.1880.10825889.

View This Item Online: https://www.biodiversitylibrary.org/item/8650

DOI: https://doi.org/10.1080/00378941.1880.10825889

Permalink: https://www.biodiversitylibrary.org/partpdf/157808

\section{Holding Institution}

Missouri Botanical Garden, Peter H. Raven Library

\section{Sponsored by}

Missouri Botanical Garden

\section{Copyright \& Reuse}

Copyright Status: Public domain. The BHL considers that this work is no longer under copyright protection.

This document was created from content at the Biodiversity Heritage Library, the world's largest open access digital library for biodiversity literature and archives. Visit BHL at https://www.biodiversitylibrary.org. 